\title{
Multivariable clinical-genetic model for predicting dyskinesia in early-onset Parkinson's disease
}

\author{
Yong-Ping Chen, Ru-Wei Ou, Xiao-Jing Gu, Ling-Yu Zhang, Bei Cao, Yan-Bing Hou, Kun-Cheng Liu, Jun-Yu Lin, \\ Qian-Qian Wei, Bi Zhao, Ying Wu and Hui-Fang Shang ${ }^{*}$ (D)
}

\begin{abstract}
Main text
The levodopa-induced dyskinesias (LIDs) in Parkinson' disease (PD) patients during levodopa treatment can lead to significant disability. Accumulative evidence has suggested that the younger the age of onset, the more likely the development of LIDs [1]. Till now, most of the studies on clinical or genetic risk factors for LIDs were cross-sectional [2], or included limited sample size [3], or mainly included late-onset PD patients [4]. Here, we investigated the incidence of LIDs in the early stage of early-onset PD (EOPD), including the first 5 years of duration and the first 5 years of dopamine replacement therapy (DRT), and established and validated clinicalgenetic models for LID prediction. Detailed methods are provided in Supplementary File 1.
\end{abstract}

A total of 279 EOPD patients with age at onset $<50$ years were included as the derivation cohort (Table S1), and 99 of them developed LIDs during follow-up at the time of analysis, including peak-dose dyskinesias (68.7\%), diphasic dyskinesias (7.1\%) and "off"-state dystonia (27.3\%). The detailed flowchart of this study, including the derivation cohort and the validation cohort, is shown in Fig. S1. Eleven single nucleotide polymorphisms (SNPs), as the candidate genetic predictors, are shown in Table S2. The levodopa equivalent daily dose (LEDD) was significantly different between LID and non-LID groups at baseline $(P<0.001)$. A total of 43 EOPD patients developed LIDs (15.4\%) in the first 5 years of duration, and 56 patients developed LIDs after over 5 years of disease duration; and the former patients

\footnotetext{
* Correspondence: hfshang2002@126.com

Department of Neurology, Laboratory of Neurodegenerative Disorders, Rare Disease Center, West China Hospital, Sichuan University, Chengdu 610041, China
}

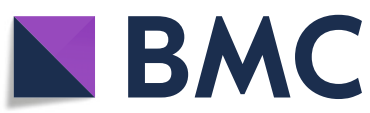

had a shorter duration at baseline than the latter ones (Table S3). In addition, of the 232 patients who received DRT for more than 5 years, 69 patients $(29.7 \%, 69 / 232)$ developed LIDs in the first 5 years of DRT. Both the incidence of LIDs at the first 5 years of duration and at the first 5 years of DRT in the derivation cohort were similar with those in the validation cohort $(n=144)(16.0 \%$ and $30.4 \%$, respectively, Table S4).

Receiver operating characteristic curves plotted using 11 clinical variables with or without genetic variables were analyzed. For LIDs at the first 5 years of duration, the area under the curve (AUC) was 0.71 (95\%CI: $0.66-$ $0.77)$ when only clinical variables were used, and increased to 0.86 (95\%CI: $0.82-0.90)(P=0.0004)$ when genotype data of 11 candidate SNPs were added (Fig. 1a). The established prediction models were confirmed in the validation group, with AUC of 0.74 (95\%CI: $0.66-$ 0.81 ) when only clinical variables were used, which increased to 0.88 (95\%CI: $0.82-0.93)(P=0.0034)$ when genotype data were added (Fig. 1b). There was no significant difference in AUC in the clinical model or clinical-genetic model between the derivation and the validation groups (Table S5). For LIDs occurring at the first 5 years of DRT, the AUC was 0.71 (95\%CI: 0.650.77 ) when only clinical variables were used, and increased to 0.80 (95\%CI: 0.74-0.85) $(P=0.0045)$ when genotype data were added (Fig. 1c). In the validation group, the AUC was 0.69 (95\%CI: 0.59 to 0.78 ) when only clinical variables were used, and increased to 0.88 (95\%CI: $0.80-0.94) \quad(P=0.0010)$ when genotype data were added (Fig. 1d). There was no significant difference in AUC in the clinical model or the clinical-genetic model between the derivation and the validation groups (Table S5). In addition, there were no significant

(c) The Author(s). 2021 Open Access This article is licensed under a Creative Commons Attribution 4.0 International License, which permits use, sharing, adaptation, distribution and reproduction in any medium or format, as long as you give appropriate credit to the original author(s) and the source, provide a link to the Creative Commons licence, and indicate if changes were made. The images or other third party material in this article are included in the article's Creative Commons licence, unless indicated otherwise in a credit line to the material. If material is not included in the article's Creative Commons licence and your intended use is not permitted by statutory regulation or exceeds the permitted use, you will need to obtain permission directly from the copyright holder. To view a copy of this licence, visit http://creativecommons.org/licenses/by/4.0/ The Creative Commons Public Domain Dedication waiver (http://creativecommons.org/publicdomain/zero/1.0/) applies to the data made available in this article, unless otherwise stated in a credit line to the data. 


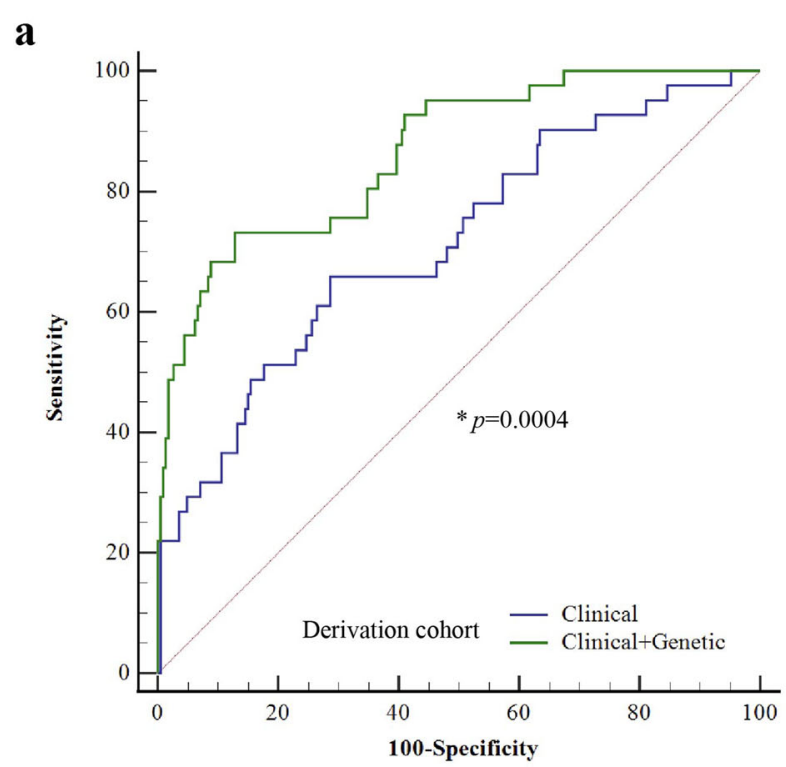

b
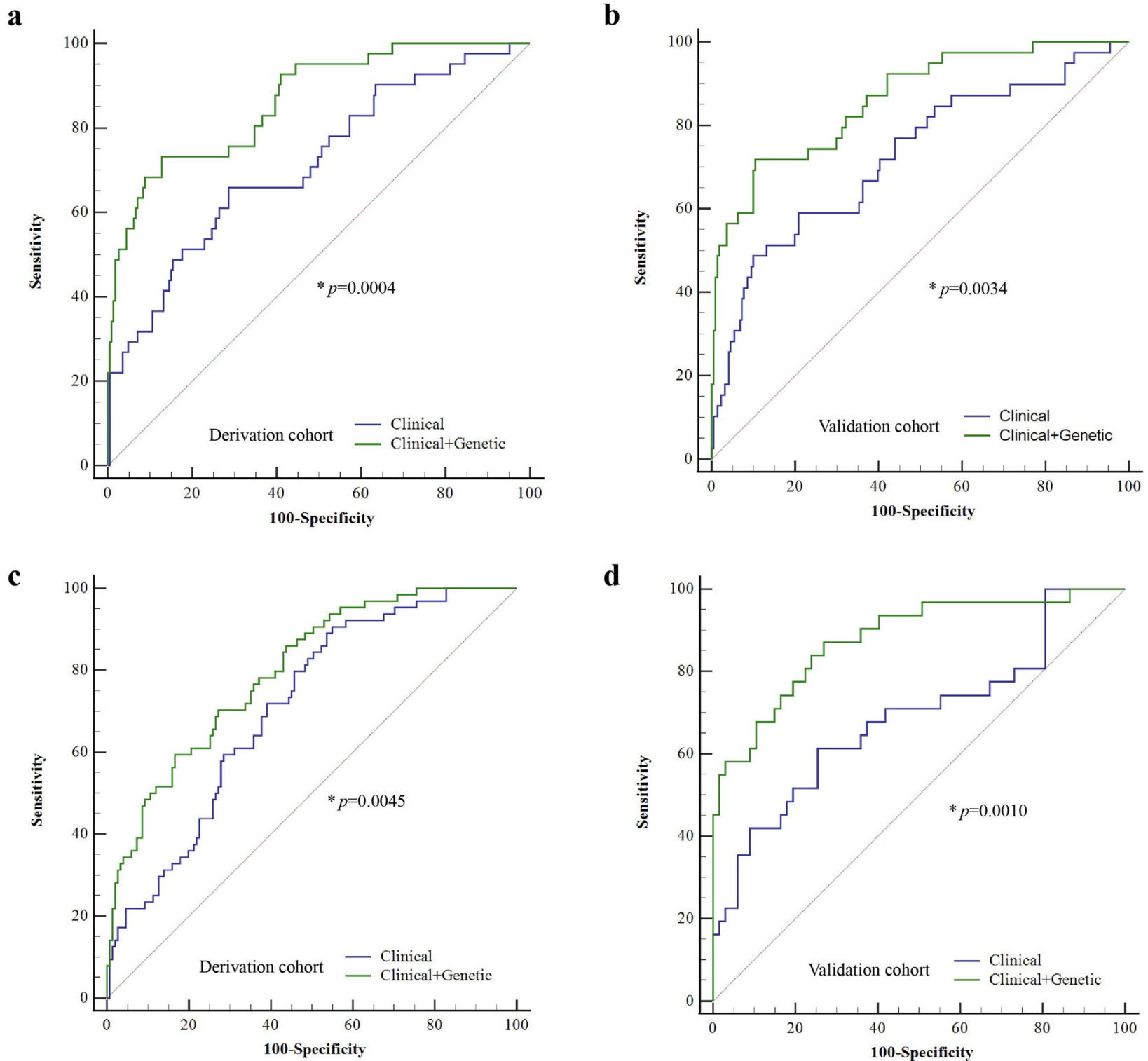

Fig. 1 ROC curves for prediction of LID incidence during the first 5 years of duration $(\mathbf{a}, \mathbf{b})$ and during the first 5 years of DRT (c, d), using two different models in the derivation $(\mathbf{a}, \mathbf{c})$ and the validation groups $(\mathbf{b}, \mathbf{d})$. Clinical variables included sex, onset age, duration, initial treatment, LEDD, initial symptoms, BMI, UPDRS-III, H\&Y staging, hyposmia, and family history. Genetic variables included genotype data on 11 candidate SNPs. a Clinical model AUC $=0.714,95 \% \mathrm{Cl}, 0.655-0.767$; clinical-genetics model $\mathrm{AUC}=0.864,95 \% \mathrm{Cl}, 0.817-0.903 ; n=279$. $\mathbf{b}$ Clinical model $\mathrm{AUC}=$ $0.740,95 \% \mathrm{Cl}, 0.657-0.811$; clinical-genetics model $\mathrm{AUC}=0.884,95 \% \mathrm{Cl}, 0.817-0.932 ; n=144$. c Clinical model $\mathrm{AUC}=0.710,95 \% \mathrm{Cl}, 0.645-0.770$; clinical-genetics model AUC $=0.798,95 \% \mathrm{Cl}, 0.738-0.850 ; n=232$. $\mathbf{d}$ Clinical model AUC $=0.687,95 \% \mathrm{Cl}, 0.585-0.777$; clinical-genetics model $\mathrm{AUC}=$ $0.879,95 \% \mathrm{Cl}, 0.798-0.936 ; n=102$

differences in AUCs for LID incidence during the first 5 years of duration or during the first 5 years of DRT, based on either model, between the derivation group and the subgroups excluding patients with PD-causative gene mutations (Table S6).

Backward stepwise regression showed that for LID incidence at the first 5 years of duration, the clinical predictors included LEDD and disease duration, and the genetic predictors included DRD2, DRD3, SLC6A3,
HRAS, COMT and BDNF genotypes. Further multivariate logistic regression analysis showed that LEDD at baseline was significantly associated with LID incidence $(P=3.4 \mathrm{e}-7)$. The minor homozygous genotype of DRD3 rs6280 increased LID incidence $(P=0.001, \mathrm{OR}=7.2)$, while the heterozygous genotype of HRAS rs12628 significantly decreased LID incidence $(P=0.005, \mathrm{OR}=0.22)$ (Table S7). For LID incidence during the 5 years of DRT, LEDD significantly increased the risk of LIDs $(P=1.7 \mathrm{e}-4)$. 
In our cohort, the incidence of LIDs was $15.4 \%$ in the first 5 years of duration, and increased to $29.7 \%$ in the first 5 years of DRT. In a previous study, the incidence of LIDs has been reported to be $21.7 \%$ in Chinese LOPD patients [5], suggesting that the LIDs are common in EOPD, and EOPD patients are susceptible to the development of LIDs. Importantly, the AUCs in the clinical or clinical-genetic model for predicting LID incidence remained consistent after excluding patients carrying PD causative gene variants, suggesting that the established clinical-genetic model is potentially useful for prediction of LIDs in all EOPD patients. In addition, the multivariate logistic regression results on LEDD are consistent with the recent finding that LEDD is the most strong clinical variable for predicting LIDs [4] and with the recommendation of a low initial dosage of LEDD for PD in China [6]. The DRD3 rs6280 GG genotype, as the strongest genetic variable, increased the risk of LIDs compared with the AA genotype in our cohort, which was consistent with the finding from a Korean cohort [7]. The distinct responses to levodopa may be attributed to the lower affinity of the AA genotype to dopamine [8].

In conclusion, the established clinical-genetic models and the identified independent predictors in the early stage of EOPD in Chinese patients may have good application prospects for LID prediction and DRT management.

\section{Abbreviations}

PD: Parkinson's disease; EOPD: Early-onset PD; LOPD: Late-onset PD;

LIDs: Levodopa-induced dyskinesias; DRT: Dopamine replacement therapy

\section{Supplementary Information}

The online version contains supplementary material available at https://doi. org/10.1186/s40035-021-00251-4

\section{Additional file 1.}

Additional file 2: Table S1. Baseline characteristics of patients in the derivation cohort. Table S2. Characteristics of the selected genetic variants. Table S3. Baseline characteristics of patients with LIDs in the derivation cohort. Table S4. Baseline characteristics of patients in the validation cohort. Table S5. Comparison of different prediction models between the derivation group and the validation group. Table S6. Comparison of different prediction models between the derivation group and subgroups excluding PD-causative gene mutations. Table S7. Single-factor association with LID incidence in the multivariable nonlinear models. Fig. S1. Flowchart of the study.

\section{Acknowledgements}

The authors appreciate all cohort individuals and their families for their participation in this study.

\section{Authors' contributions}

Y-PC: Study design, blood sample collection, DNA extraction, data analysis and writing. R-WO: Clinical data collection. X-JG: Blood sample collection and DNA extraction, variants filtration. L-YZ: Clinical data collection. BC: Patient enrollment and clinical data collection. Y-BH: Clinical data collection. K-CL: Clinical data collection. J-YL: Clinical data collection. Q-OW: Clinical data collection. BZ: Patient enrollment. YW: Patient enrollment. H-FS: Conception, organization, review and critique. The authors read and approved the final manuscript.

\section{Funding}

This study was supported by the National Key Research and Development Program of China (2018YFC1312001 and 2016YFC0901504), the National Natural Science Foundation of China (81971188) and the 1.3 .5 project for Disciplines of Excellence, West China Hospital, Sichuan University (ZYJC18038, ZYJC18003 and 2019HXFH046).

\section{Availability of data and materials}

The datasets used and/or analyzed during the current study are available from the corresponding author on reasonable request.

\section{Declarations}

Ethics approval and consent to participate

All participants had given informed consent to participate and the study was approved by the ethics committee of West China Hospital, Sichuan

University.

\section{Consent to publication}

Not applicable.

\section{Competing interests}

The authors report no conflicts of interest.

Received: 15 June 2021 Accepted: 15 July 2021

Published online: 29 July 2021

References

1. Ku S, Glass GA. Age of Parkinson's disease onset as a predictor for the development of dyskinesia. Mov Disord. 2010;25(9):1177-82.

2. Schrag A, Quinn N. Dyskinesias and motor fluctuations in Parkinson's disease. A community-based study. Brain. 2000;123(Pt 11):2297-305.

3. Bjornestad A, Forsaa EB, Pedersen KF, Tysnes OB, Larsen JP, Alves G. Risk and course of motor complications in a population-based incident Parkinson's disease cohort. Parkinsonism Relat Disord. 2016;22:48-53.

4. Eusebi P, Romoli M, Paoletti FP, Tambasco N, Calabresi P, Parnetti L. Risk factors of levodopa-induced dyskinesia in Parkinson's disease: results from the PPMI cohort. NPJ Parkinsons Dis. 2018;4(1):33.

5. Chen W, Xiao Q, Shao M, Feng T, Liu WG, Luo XG, et al. Prevalence of wearing-off and dyskinesia among the patients with Parkinson's disease on levodopa therapy: a multi-center registry survey in mainland China. Transl Neurodegener. 2014;3(1):26.

6. Chen S, Chan P, Sun S, Chen H, Zhang B, Le W, et al. The recommendations of Chinese Parkinson's disease and movement disorder society consensus on therapeutic management of Parkinson's disease. Transl Neurodegener. 2016;5(1):12

7. Lee JY, Cho J, Lee EK, Park SS, Jeon BS. Differential genetic susceptibility in diphasic and peak-dose dyskinesias in Parkinson's disease. Mov Disord. 2011 26(1):73-9.

8. Steen VM, Løvlie R, MacEwan T, McCreadie RG. Dopamine D3-receptor gene variant and susceptibility to tardive dyskinesia in schizophrenic patients. Mol Psychiatry. 1997;2(2):139-45.

Ready to submit your research? Choose BMC and benefit from:

- fast, convenient online submission

- thorough peer review by experienced researchers in your field

- rapid publication on acceptance

- support for research data, including large and complex data types

- gold Open Access which fosters wider collaboration and increased citations

- maximum visibility for your research: over $100 \mathrm{M}$ website views per year

At BMC, research is always in progress.

Learn more biomedcentral.com/submissions 